ARTIGO ORIGINAL

ISSN 1677-5090

(C) 2018 Revista de Ciências Médicas e Biológicas

DOI: http://dx.doi.org/10.9771/cmbio.v17i3.28628

\title{
Avaliação radiográfica da interface de reparo em resina bulk fill
}

\author{
Radiographic evaluation of repair interface in bulk fill resin
}

\author{
Ana Paula Araujo Teixeira ${ }^{1}$, Paula Mathias de Moraes Canedo ${ }^{2 *}$, Rafael Soares Gomes ${ }^{3}$ \\ ${ }^{1}$ Mestranda em Processos Interativos de Órgãos e Sistemas pelo ICS/UFBA.;2 Docente da da Faculdade de \\ Odontologia da UFBA.; ${ }^{3}$ Mestre e Doutorando em Clínica Odontológica - Prótese Dental pela FOP/UNICAMP
}

\begin{abstract}
Resumo
Objetivo: avaliar radiograficamente a interface de reparo em resina bulk fill (Opus bulk fill - FGM) com diferentes protocolos de adesão. Metodologia: foram confeccionados 30 corpos de prova em formato de cubo, nas dimensões de $4 \mathrm{~mm} \times 6 \mathrm{~mm}$. Os corpos foram randomizados e divididos entre os grupos: G1: ácido fosfórico a 37\% (Dentsply) + adesivo (Ambar - FGM); G2: ácido fosfórico a 37\% (Dentsply) + silano (Prosil - FGM) + adesivo (Ambar-FGM) e G3: Adesivo auto-condicionante (Ambar universal - FGM). Após os tratamentos, os corpos foram posicionados em uma matriz metálica de $4 \mathrm{~mm} \times 8 \mathrm{~mm}$ para receber novos incrementos de resina bulk fill. Para análise do reparo, as amostras foram submetidas a radiografias digitais (Micro Imagem), com disparo de 0,16 segundos. Aplicou-se o efeito de contraste filtro de tele MI para permitir a avaliação visual. Para análise estatística foi utilizado o teste exato de Fisher. Resultados: o grupo 2 apresentou em metade dos espécimes avaliados, uma interface não visível, porém, não diferiu estatisticamente dos outros grupos que em sua maioria apresentaram a interface visível em mais de $50 \%$ da extensão do reparo. Conclusão: os protocolos realizados na interface da resina bulk fill não foram suficientes para gerar diferenças entre as imagens dos grupos tratados.
\end{abstract}

Palavras chave: Resinas. Restauração dentária permanente. Reparação de Restauração dentária.

\begin{abstract}
Objective: evaluate radiographically repair interface in bulk fill resin (Opus bulk fill-FGM) with different protocols of adhesion. Metodology: 30 specimens on the dimensions of $4 \mathrm{~mm} \times 6 \mathrm{~mm}$ were made in cube formate. The specimens were randomized and divided between the groups: G1: phosphoric acid at 37\% (Dentsply) + sticker (Ambar-FGM), G2: phosphoric acid at 37\% (Dentsply) + silane (Prosil-FGM) + sticker (Ambar-FGM) and G3: Self-etching adhesive (universal Amber-FGM). After the treatments, the specimens were placed in a $4 \mathrm{~mm} \times 8 \mathrm{~mm}$ metal matrix to receive new increments of resin bulk fill. For the repair analysis, samples were submitted to digital $x$-rays (Micro image), with triggering of 0.16 seconds. The filter contrast effect tele filter $\mathrm{MI}$ to allow visual assessment was applied. For statistical analysis we used the Fisher exact test. Results: group 2 presented in half of the specimens evaluated a not visible interface, however did not differ statistically from the other groups in that in their majority presented the visible interface in more than $50 \%$ of the repair extent. Conclusion: the protocols carried out in bulk fill resin interface were not sufficient to generate differences between the images of the treated groups.
\end{abstract}

Keywords: Resins. Permanent dental restoration. Dental restoration repair.

\section{INTRODUÇÃO}

A busca pelo material restaurador ideal vem sendo cada vez mais constante na Odontologia. Propriedades químicas e físicas de materiais são analisadas frequentemente, a fim de proporcionar resistência, longevidade, biocompatibilidade, adesão e estética às restaurações e melhor atender à demandas dos pacientes (LEPRINCE, 2014). As resinas compostas são materiais utilizados em grande escala, e, como tal, estão sujeitas a pesquisas e a estudos comparativos para a obtenção dos melhores resultados, o que, consequentemente, tem implicações na aplicabilidade clínica.

Diante da gama de materiais restauradores, a resina composta tem mostrado resultados satisfatórios, e, por-

Correspondente/Corresponding: *Paula Mathias de Moraes Canedo - Faculdade de Odontologia, Universidade Federal da Bahia - End: Av. Araújo Pinho, 72, Canela, Salvador, Ba. CEP: 40.110-912 - Tel: (71) 99116-7275 -E-mail: pmathias@yahoo.com tanto, seu uso é preconizado em restaurações diretas de dentes anteriores e posteriores (FERRACANE, 2011; RUEGGEBERG, 2002). Contudo, há o comprometimento da longevidade desse material, por conta dos desafios que ocorrem na cavidade oral, a exemplo do manchamento, degradação mecânica e hidrolítica, além de desgastes (OPDAM, et al., 2014).

Nessa perspectiva, as restaurações são submetidas a constantes trocas, culminando no comprometimento de estruturas dentárias sadias, pois, ao se realizar a remoção completa do material, há a necessidade de ampliar a cavidade. A esse fato pode-se atribuir a diminuição das propriedades mecânicas, bem como, o comprometimento do complexo dentinopulpar, reduzindo-se dessa forma a sobrevida da unidade dentária, além de se aumentarem os custos e o tempo de trabalho (BLUM; JAGGER; WILSON, 2011).

A odontologia minimamente invasiva preconiza reparos em restaurações de resina, desde que haja 
defeitos parciais como pigmentações, falhas marginais, rugosidade, fraturas e anatomia insatisfatória. Os reparos consistem na remoção da resina ou de qualquer tecido subjacente defeituoso associada a reconstrução da estrutura restaurada (HICKEL; BRÜSHAVER; ILIE, 2013). Utilizando-se a técnica do reparo, o procedimento será conservador e trará resultados satisfatórios, com baixo custo, menor tempo clínico e longevidade da restauração (BLUM; LYNCH; WILSON, 2012; HICKEL; BRÜSHAVER; ILIE, 2013). Deve-se considerar, como fatores limitantes do reparo, o risco de cárie que deve ser avaliado em cada paciente, os hábitos parafuncionais, a oclusão, tamanho e profundidade da cavidade (FERNÁNDEZ et al., 2015a).

Sendo o reparo, um procedimento clínico rotineiro no consultório, é necessário considerar que, futuramente, ele será realizado com resinas bulk fill, já que elas entraram no mercado recentemente e possuem propriedades físicas e químicas tão satisfatórias quanto as resinas convencionais. As resinas bulk fill surgiram no mercado odontológico com a proposta de uma técnica na qual a polimerização ocorre em profundidade de até $5 \mathrm{~mm}$, sem alterações na qualidade da restauração, já que elas, possuem menor contração de polimerização, se comparadas às resinas convencionais (ALQAHTANI et al., 2015; FURNESS et al., 2014).

Diante do exposto, é necessário verificar as possibilidades de reparo em resinas bulk fill, tendo em vista que seu uso tem sido cada vez mais constante na Odontologia. Do mesmo modo que os outros materiais resinosos, as resinas bulk fill também sofrem modificações ao longo do tempo, e, portanto, pressupõe-se que o reparo poderá ser um procedimento vantajoso para a preservação da estrutura dentária. Este estudo tem como objetivo avaliar radiograficamente a interface de reparo em resina bulk fill submetida a diferentes protocolos adesivos.

\section{METODOLOGIA}

\section{SELEÇÃO DO MATERIAL}

Para a confecção dos corpos de prova do presente estudo, foi selecionada a resina Opus bulk fill de consistência regular (FGM Produtos Odontológicos, Joinville, SC). Foram constituídas 30 amostras, que foram distribuídas em três grupos para análise radiográfica da interface de reparo. Os grupos foram divididos de acordo o protocolo adesivo utilizado. G1: Ácido fosfórico a 37\% (Densply, Pirassununga-SP) + adesivo (Ambar-FGM Produtos Odontológicos, Joinville, SC); G2: Ácido fosfórico a 37\% (Dentsply, Pirassununga - SP) + Silano (Prosil-FGM Produtos Odontológicos, Joinville, $\mathrm{SC}$ ) +adesivo (AmbarFGM Produtos Odontológicos, Joinville, SC) e G3: adesivo auto-condicionante (Ambar Universal - FGM Produtos Odontológicos, Joinville, SC). A composição dos materiais encontra-se na Tabela 1.

Tabela 1 - Composição dos materiais

\begin{tabular}{|c|c|c|}
\hline MATERIAIS & CLASSIFICAÇÃO & COMPOSIÇÃO \\
\hline Opus Bulk Fill (FGM) & $\begin{array}{l}\text { Resina composta fotopolimerizável de } \\
\text { baixa tensão de polimerização. }\end{array}$ & $\begin{array}{l}\text { Ingredientes ativos: Monômeros uretanadimetacrílicos, estabilizantes,fotoiniciadores } \\
\text { e co-iniciadores. } \\
\text { Ingredientes inativos: Carga inorgânica de dióxido de silício (sílica) silanizado, } \\
\text { estabilizantes e pigmentos. }\end{array}$ \\
\hline Ácido fosfórico a 37\% (Dentsply) & Condicionador dental & Ácido Fosfórico; Surfactante; Aerosil 200; Água deionizada e Pigmento. \\
\hline \multirow[t]{2}{*}{ 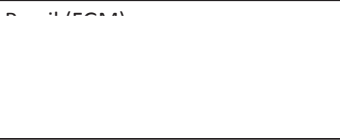 } & $\cdot \cdot$ & Solução etanólica de 3-Metacriloxipropiltrimetoxisilano hidrolisada \\
\hline & & $\begin{array}{l}\text { Ingredientes ativos: MDP (10-metacriloiloxidecil dihidrogênio fosfato), monômeros } \\
\text { metacrílicos, composição fotoiniciadora (APS), co-iniciadores e estabilizantes. } \\
\text { Ingredientes inativos: Carga inerte (nanopartículas de sílica) e veículo (etanol). }\end{array}$ \\
\hline Ambar Universal (FGM) & $\begin{array}{l}\text { Sistema adesivo fotopolimerizável } \\
\text { convencional de dois passos. } \\
\text { 7ạ geração }\end{array}$ & $\begin{array}{l}\text { Ingredientes ativos: MDP (10-Metacriloiloxidecil dihidrogênio fosfato), } \\
\text { monômeros metacrílicos, fotoiniciadores, coiniciadores e estabilizante. } \\
\text { Ingredientes inativos: Carga inerte (nanopartículas de sílica) e veículo (etanol). }\end{array}$ \\
\hline
\end{tabular}

Fonte: Autoria própria

\section{OBTENÇÃO DOS CORPOS DE PROVA}

A confecção das amostras foi realizada em três etapas. Inicialmente, selecionou-se uma matriz metálica bipartida quadrada de bordas nítidas, na dimensão de 4 $\mathrm{mm}$ de altura, $6 \mathrm{~mm}$ de largura e $6 \mathrm{~mm}$ de comprimento. A matriz foi posicionada em uma placa de vidro, e em seguida, a resina foi inserida em um único incremento de $4 \mathrm{~mm}$, com uma espátula de inserção $\mathrm{n}$ ㅇ 1(Suprafill Duflex SSWhite, Rio de Janeiro, RJ). Removeram-se os excessos e logo após, uma tira de poliéster foi sobreposta à resina para promover a lisura superficial. Um peso de 50 gramas pressionou a estrutura por 30 segundos. A polimerização foi feita com LED (VALO, Ultradent Products Inc., South Jordan, UT, USA), por 40 segundos e foi realizada uma polimerização adicional de 40 segundos, após a remoção dos corpos da matriz. Os corpos de prova foram imersos em água deionizada e armazenados em estufa a 370.

$\mathrm{Na}$ segunda etapa, as amostras foram divididas aleatoriamente e receberam os tratamentos propostos para cada grupo. Em todos os grupos seguiram-se as recomendações dos fabricantes 
Figura 1 - A) Matrizes B) Inserção da resina C) Polimerização
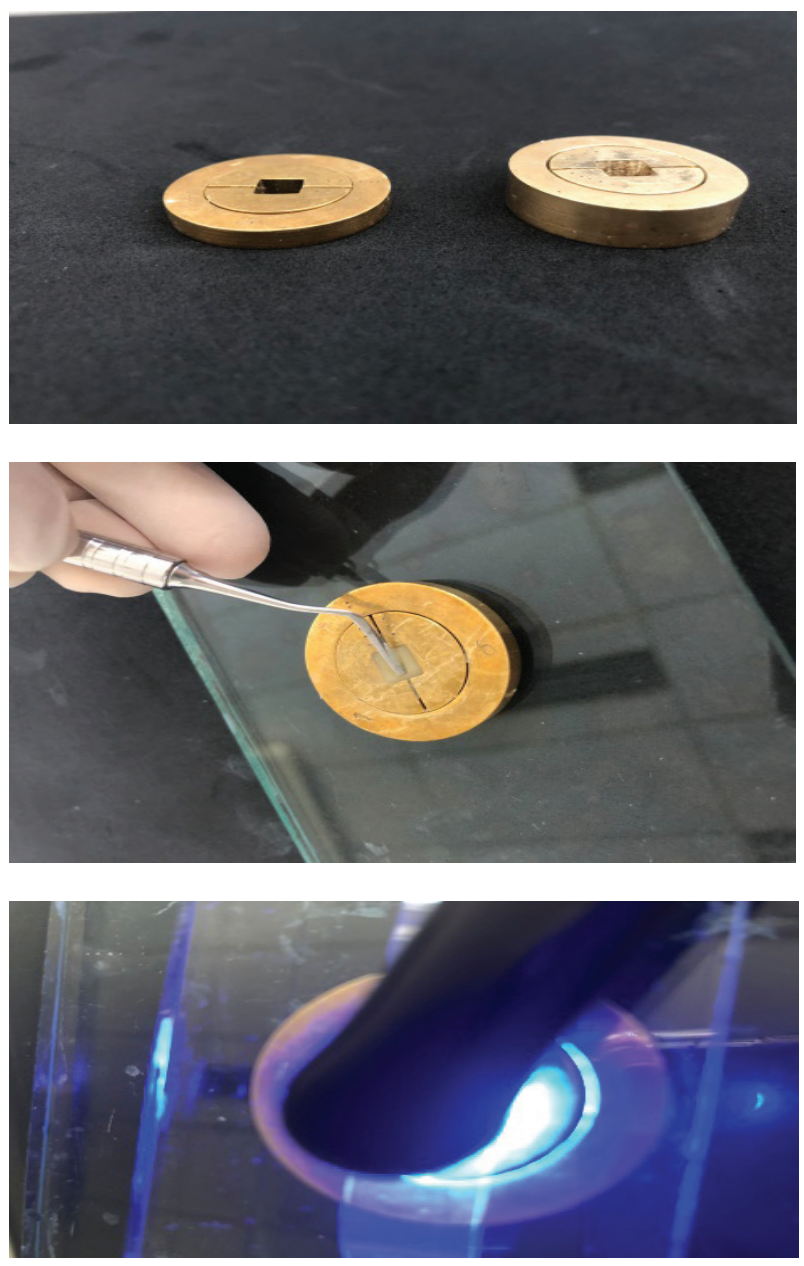

Fonte: Autoria própria

\section{G1: Ácido fosfórico +Adesivo}

Realizou-se o condicionamento com ácido fosfórico a $37 \%$ (Densply, Pirassununga, SP) durante 30 segundos, e a superfície foi lavada abundantemente e seca com papel absorvente. Com um aplicador (Kavibrush, FGM, Joinville, SC) foi aplicada a primeira camada do adesivo, friccionando vigorosamente por 10 segundos; a segunda camada do produto foi aplicada em seguida, por mais 10 segundos, e leves jatos de ar foram dispensados para promover a evaporação do solvente. A fotopolimerização (VALO - Ultradent Products Inc., South Jordan, UT, USA) foi feita por 10 segundos.

\section{G2: Ácido fosfórico a 37\% + silano + adesivo}

Anteriormente à silanização, houve o condicionamento com ácido fosfórico a 37\% (Dentsply, Pirassununga-SP), durante 30 segundos $A$ superfície resinosa foi lavada abundantemente e seca com papel absorvente. Aplicou- -se uma fina camada do silano (Prosil-FGM, Joinville, SC) e, após um minuto foram dispensados jatos de ar. Observou-se o brilho da superfície e fez-se a aplicação do adesivo (Ambar-FGM, Joinville - SC) em duas camadas. A primeira foi aplicada com fricção vigorosa por 10 segundos; logo após, a segunda camada foi dispensada por espalhamento, e jatos de ar foram aplicados à distância para evaporação do solvente, prosseguindo com a polimerização (VALO - Ultradent Products Inc., South Jordan, UT, USA) por 10 segundos.

\section{G3: Adesivo autocondicionante}

Não há necessidade de condicionamento ácido nesta variação. Portanto, realizou-se a aplicação da primeira camada do adesivo com aplicador descartável (Kavibrush, FGM, Joinville - SC), friccionando-se vigorosamente durante 10 segundos, em seguida a segunda camada foi aplicada espalhando o produto. Utilizaram-se jatos de ar à distância para evaporação do solvente. Seguindo-se o protocolo, houve a polimerização (VALO - Ultradent Products Inc., South Jordan, UT, USA) com LED, por 10 segundos.

Após o condicionamento adesivo dos três grupos, procedeu-se à terceira etapa da confecção das amostras. Os corpos de prova foram posicionados em uma matriz metálica quadrada nas dimensões $4 \times 8$, para haver a complementação em incremento único com resina bulk fill. Após a inserção da resina, foi inserida uma tira de poliéster e um peso foi sobreposto durante 30 segundos. A polimerização foi realizada durante 40 segundos (VALO - Ultradent Products Inc., South Jordan, UT, USA). Os excessos foram removidos com lâmina de bisturi no 12 .

\section{AVALIAÇÃO RADIOGRÁFICA DA INTERFACE DE REPARO}

Para a análise da interface de reparo, realizaram-se radiografias em aparelho digital (Micro Imagem, Indaiatuba,SP,Brasil), através do sensor para radiografia digital micro imagem -FIT -T2, com disparo de 0,16 segundos (modelo 6802.0616499). Após a tomada radiográfica dos três grupos - G1; G2 e G3, aplicou-se o efeito de contraste filtro de tele $\mathrm{MI}$ para uma visualização mais fidedigna da linha de reparo.

A classificação foi feita através do score apresentado na Tabela 2:

Tabela 2 - Score da avaliação radiográfica

\begin{tabular}{l}
\hline 0 - Interface não visível \\
1 - Interface visível em menos de $50 \%$ \\
2 - Interface visível em mais de $50 \%$ \\
3 - Interface visível em $100 \%$ de sua extensão
\end{tabular}

Fonte: Autoria própria 
Figura 2-Score 0

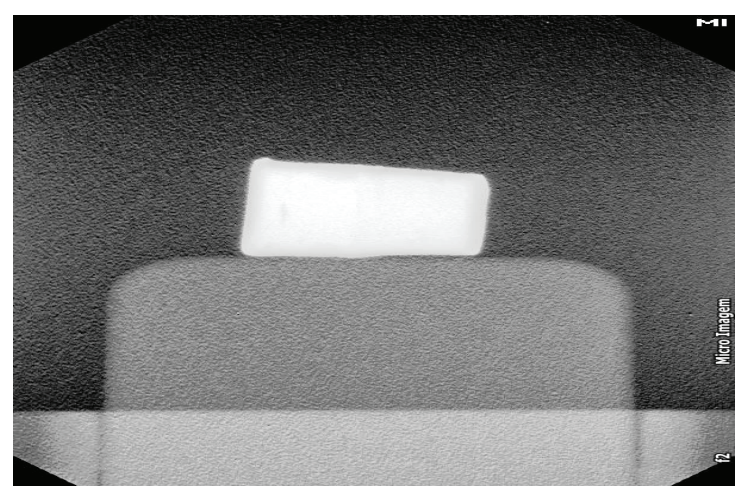

Figura 3 - Score 1

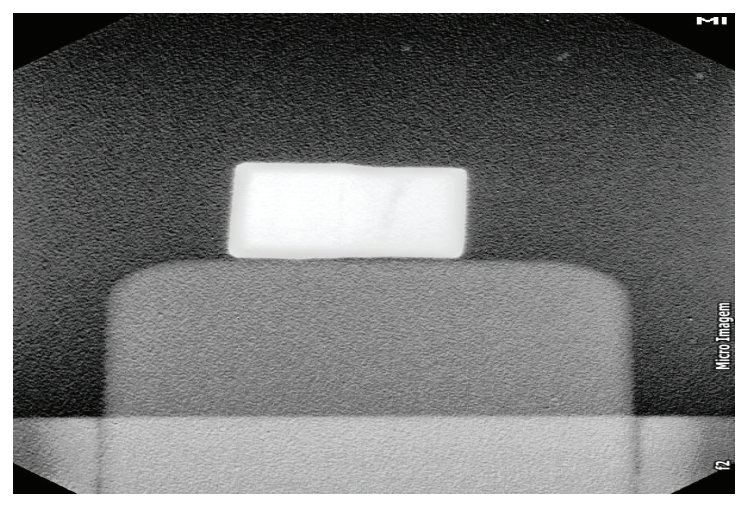

Figura 4 - Score 2

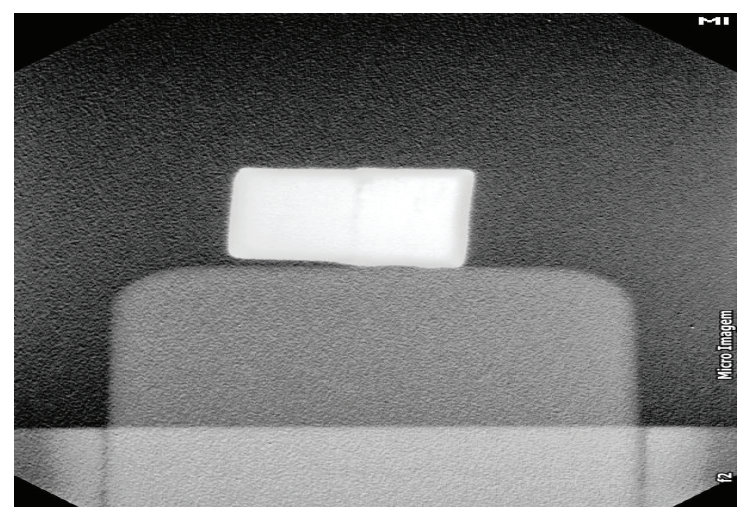

Figura 5 - Score 3

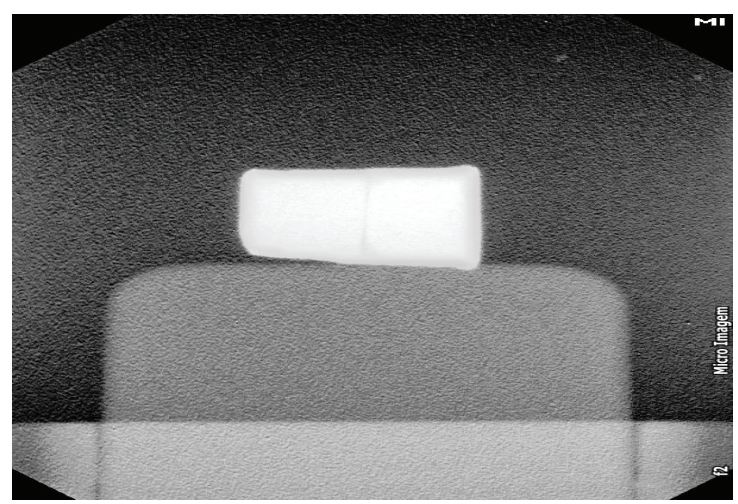

Fonte: Autoria própria

\section{ANÁLISE ESTATÍSTICA}

Para a análise estatística, os dados foram alocados em uma tabela de contingência $3 \times 4$, sendo a variável grupo adotada como nominal e a visibilidade da interface como uma variável categórica ordinal. Devido à presença de valores esperados menores que 5 , foi utilizado o teste Exato de Fisher. Os dados estão apresentados no quadro a seguir:

Quadro 1-Tabulação cruzada Visibilidade da Interface * Grupos

\begin{tabular}{|c|c|c|c|c|c|}
\hline & & \multicolumn{3}{|c|}{ Grupos } & \multirow[b]{2}{*}{ Total } \\
\hline & & Ambar & $\begin{array}{l}\text { Ambar } \\
+ \text { Prosil }\end{array}$ & $\begin{array}{c}\text { Ambar } \\
\text { Universal }\end{array}$ & \\
\hline \multirow{4}{*}{$\begin{array}{l}\text { Visibilidade } \\
\text { da interface }\end{array}$} & Interface não visível & $3_{a}$ & 5 & 2 & 10 \\
\hline & $\begin{array}{l}\text { Interface visível em } \\
\text { menos de } 50 \%\end{array}$ & $2 a$ & $3_{a}$ & $3_{a}$ & 8 \\
\hline & $\begin{array}{l}\text { Interface visível em } \\
\text { mais de } 50 \%\end{array}$ & $4_{a}$ & $1_{\mathrm{a}}$ & $5_{a}$ & 10 \\
\hline & $\begin{array}{l}\text { Interface visível em } \\
100 \% \text { da extensão }\end{array}$ & $1_{a}$ & $1_{\mathrm{a}}$ & $0_{a}$ & 2 \\
\hline Total & & 10 & 10 & 10 & 30 \\
\hline
\end{tabular}

Fonte: Autoria própria

\section{RESULTADOS}

Ao realizar a análise da linha de reparo nos Grupos G1,G2, e G3, observou-se que no Grupo 1 prevaleceu o score 2 (interface visível em mais de 50\%), no G2 observou-se em sua maioria o score 0 (interface não visível) e no $\mathrm{G} 3$ o resultado foi similar ao G1 (interface visível em mais de $50 \%)$.

Figura 6 - Distribuição da visibilidade da interface dos grupos de estudo.

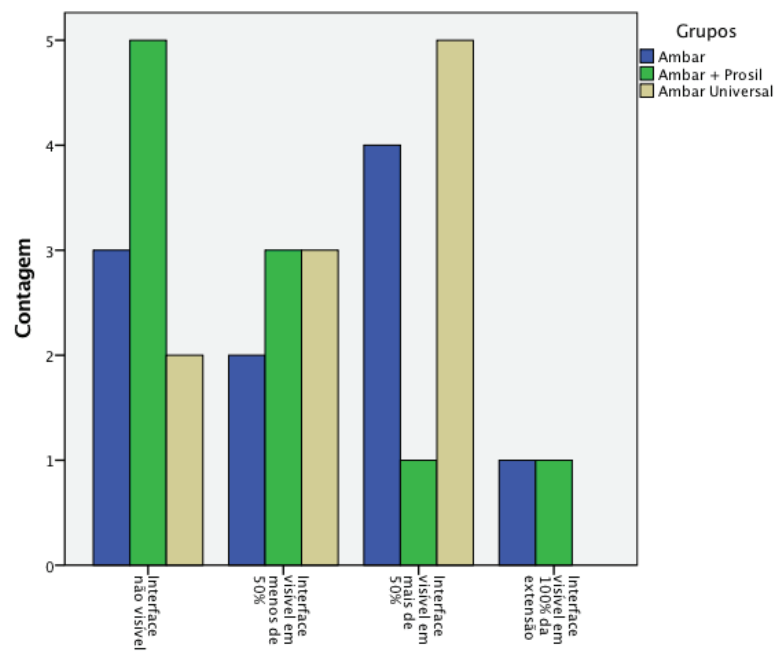

Fonte: Autoria própria

\section{DISCUSSÃO}

Dentre as técnicas para o tratamento de superfície mais recomendadas, estão os protocolos adesivos e o aumento da rugosidade com pontas diamantadas. Não 
há um consenso, na literatura, sobre a melhor técnica. Entretanto, estudos comprovam que a asperização (com pontas diamantadas, ou jateamento com partículas de óxido de alumínio), bem como a utilização de um sistema adesivo, aumentam a resistência de união do reparo (HEMADRI et al., 2014; OZCAN et al, 2013). Neste estudo, optou-se pela aplicação de diferentes agentes de união intermediários em uma classe de resina, para avaliar sua eficácia quando utilizados em reparos.

Gordan et al. (2006), avaliaram a eficácia dos reparos em restaurações que apresentaram defeitos parciais. Foram analisadas 88 restaurações de resina composta com integridade marginal afetada. Elas foram divididas, em 05 grupos com diferentes tratamentos: reparo, selamento, remodelação, substituição e grupo controle. A remodelação é uma técnica utilizada em áreas superficialmente manchadas e consiste em suavizar o manchamento, nas faces interproximais, com tiras de lixa de óxido de alumínio e, nas faces livres com discos e pontas para acabamento e polimento. Concluiu-se que os grupos submetidos ao reparo e à substituição apresentaram resultados superiores aos demais. Dessa forma, mostrou-se que o reparo além de possuir caráter conservador, é uma indicação viável para sanar defeitos marginais.

Fernández et al. (2011) realizaram um estudo similar ao supracitado, embora, fosse um experimento realizado in vivo, sendo analisadas 208 restaurações que passaram pelos tratamentos de selamento marginal, remodelação e reparo. Os tratamentos foram observados durante quatro anos, bem como, avaliados segundo os critérios Ryge modificados (adaptação marginal, cáries secundárias, rugosidade, brilho e anatomia). Observou-se que as restaurações que passaram pelo tratamento de reparo obtiveram tempo médio de sobrevida influenciando positivamente na longevidade.

Em 2015, Fernández et al.(2015b) analisaram restaurações de resina composta de acordo os critérios Ryge-USPHS em 28 pacientes e 50 restaurações, num acompanhamento com duração de 10 anos. Observaram que os reparos são eficazes e contribuem para a longevidade da restauração quando feitos por indicações adequadas.

No presente estudo, o grupo 2 que recebeu o tratamento com ácido fosfórico a $37 \%$, silano e adesivo, apresentou resultado mais satisfatórios, já que, a linha de reparo na maioria dos corpos de prova não foi visível na análise radiográfica. 0 grupo 1 , tratado com ácido fosfórico a $37 \%$, associado ao adesivo, e o grupo 3 , tratado com adesivo universal autocondicionante (adesivo que contem silano), apresentaram resultados similares quanto à visualização radiográfica, evidenciando mais de $50 \%$ da linha de reparo visível. Nessa perspectiva, em 1997, Brosh et al. avaliaram o efeito de diferentes tratamentos em resinas compostas submetidas ao cisalhamento. Utilizaram ponta diamantada, disco de carburundum, jateamento com óxido de alumínio, abrasão com bicarbonato de sódio e ácido fluorídrico, e todos foram associados com resina fluída ou silano, ou a combinação de ambos. Assim como neste estudo, em que um dos grupos tratados com silano mostrou melhor resultado, o estudo acima mencionado demonstrou que o uso da resina fluída ou a combinação de resina fluida e silano foram os métodos mais eficazes para aumentar a resistência de união.

O silano é composto de moléculas organofuncionais que possuem como função unir dois materiais. No reparo, essa substância promove a união da fase inorgânica do substrato, com a fase orgânica do material resinoso (MATINLINNA, 2004). Estudos comprovam que o silano por possuir boa capacidade de molhamento, facilita a penetração do adesivo na superfície (ILIE; BUCUTA; DRAENERT, 2013).

Madeira (2002) com o objetivo de avaliar a diferença entre tratamentos que receberam ou não condicionamento com ácido fosfórico observou que o uso desse ácido não promoveu os resultados esperados, pois a realização de retenções mecânicas foi mais efetiva do que o abrasionamento, embora não houvesse diferenças estatísticas significantes. Comparando com o estudo realizado, o condicionamento ácido não teve influência negativa nos grupos em que foi utilizado, ressaltando-se - G2 que apresentou em metade dos espécimes, uma interface invisível.

Em um estudo similar a este, Hisamatsu, Atsuda e Matsumura (2002), analisaram a efetividade do silano quando associado aos adesivos na interface entre resinas compostas. Após o teste de cisalhamento, observou-se que esse é um protocolo que pode ser recomendado para os reparos, já que, houve maior resistência de união. É válido ressaltar que, no presente estudo, dentre os grupos que foram tratados com silano, o G2 apresentou, em 50\% dos corpos de prova uma interface não visível, ao passo que o G3, onde o adesivo possui incorporação de silano, o resultado não foi superior ou igual ao G2.

$O$ adesivo Ambar universal é um sistema de dois passos. Contudo, sua composição permite que seja utilizado sem ataque ácido e, como dito anteriormente, o silano está presente em sua formulação (FGM, 2017). Neste estudo observou-se que na forma autocondicionante, o adesivo não mostrou resultados melhores, quando comparados aos outros. Em contraposição a essas implicações, Teixeira et al. (2005), observaram que os adesivos autocondicionantes foram mais eficazes. No entanto, concluíram que essa característica está vinculada ao tipo de resina utilizada no reparo. Sabe-se que a resina bulk fill é consideravelmente nova no mercado, e, por isso, mais estudos devem ser realizados.

A avaliação radiográfica foi o método de escolha para avaliar visualmente a linha de reparo em uma marca de resina bulk fill. Esse teste foi escolhido devido à necessidade de análise imediata após o reparo ter sido realizado e primordialmente por ser um teste clinicamente viável. Contudo, se observou que não foi sensível o suficiente para gerar resultados e, dessa forma, outros protocolos poderão ser estabelecidos para que novas análises sejam feitas. Testes de microtração e cisalhamento são indicados 
para se obterem valores imediatos e em longo prazo a cerca da efetividade do reparo (CAMARGO et al., 2007; RIBEIRO et al., 2013). Para avaliar a resistência adesiva, o teste de microtração é considerado padrão ouro, já que nos resultados obtidos consegue-se avaliar falhas adesivas e consequentemente diminuir a interferência dos fatores relacionados (CAMARGO et al., 2007; RIBEIRO et al., 2013).

Um estudo recente (HAN et al., 2016) avaliou através de tomografia e micro CT a adaptação marginal da resina composta submetida a diferentes tratamentos (adesivos de três passos, dois passos e autocondicionantes). Contudo, esse estudo não foi realizado em reparo, e sim em cavidade de dentes bovinos. Observou-se, ao final, que em todos os grupos existiam falhas adesivas. Sendo assim, conclui-se que dentro dos parâmetros da avaliação radiográfica, tomografia e micro CT são exames acurados para avaliar restaurações em resina e poderão ter bastante utilidade para avaliar os reparos. Embora esses exames sejam de grande importância para a pesquisa científica, a rotina clínica não permite seu uso constante para avaliação de reparos, cabendo assim, novos testes de tratamento de superfície para analisar a possibilidade de obtenção de imagens radiográficas satisfatórias.

\section{CONCLUSÃO}

Diante do exposto, concluiu-se que a utilização de diferentes protocolos de adesão em reparo de resina bulk fill não gerou diferenças na interface reparada, quando avaliadas radiograficamente.

\section{REFERÊNCIAS}

ALQAHTANI, M. Q. et al. Effect of high irradiance on depth of cure of a conventional and a bulk fill resin-based composite. Oper. Dent., Seattle, v. 40, n. 6, p. $662-672,2015$.

BLUM, I. R.; JAGGER, D. C.; WILSON, H. H. Defective dental restorations: to repair or not to repair? Part 1: direct composite restorations. SADJ, [S.I], v. 66, n.3, p. 114-118, 2011.

BLUM, I. R.; LYNCH, C. D.; WILSON, N. H. J. oral rehabil., Oxford, v. 3, n. 3, p. 210-216, 2012.

BROSH, T. et al. Effect of combinations of surface treatments and bonding agents on the bond strength of repaired composites. J. prosthet. dent., St. Louis, v.77, n.2, p.122-126, 1997.

CAMARGO, M. A. Microtensile bond test: a literature overview. Rev. Inst. Ciênc. Saúde, São Paulo, v.25, n.3, p.313-318, 2007.

FERNÁNDEZ, E. et al. Can repair increase the longevity of composite resins? Results of a 10 -year clinical trial. J. Dent., Iran, v. 43, n.2, p. 279-286, 2015a.

FERNÁNDEZ, E. et al. Sealing composite with defective margins, good care or over treatment? results of a 10 -year clinical trial. Oper. Dent., Seattle, v. 40, n.2, p.144-152, 2015b.
FERNÁNDEZ, E. M. et al. Survival rate of sealed, refurbished and repaired defective restorations: 4-year follow-up. Braz. dent j., Ribeirão Preto, v. 22, n.2, p. 134-139, 2011.

FERRACANE, J. L. Resin Composite - state of the art. Dent. mater., Washington, v. 27, n.1, p. 29-38, Jan. 2011.

FGM Produtos Odontológicos. Manual de instruções Ambar Universal. Joinville, SC, 2017.

FURNESS, S. A. et al. Effect of bulk/incremental fill on internal gap formation of bulk-fill composites. J. Dent., Iran, v. 42, n. 4, p. 439-449, 2014.

GORDAN, V. V.et al. Two-year clinical evaluation of repair versus replacement of composite restorations. J. esthet. restor. dent., Hamilton, v.18, n. 3, p.144-154, 2006.

HAN, S. H. et al. Non-destructive evaluation of an internal adaptation of resin composite restoration with swept-source optical coherence tomography and micro-CT. Dent. mater, Washington, v. 32, e1-7, 2016.

HEMADRI, M. et al. Shear bond strength of repaired composites using surface treatments and repair materials: an in vitro study. J. Int. Oral Health, India, v. 6, n. 6, p. 22-25, 2014.

HICKEL, R.; BRÜSHAVER, K.; ILIE, N. Repair of restorations -criteria for decision making and clinical recommendations. Dent. mater., Washington, v. 29, n.1, p. 28-50, 2013.

HISAMATSU, N.; ATSUDA, M.; MATSUMURA, H. Effect of silane primers and unfilled resin bonding agents on repair bond strength of a prosthodontics microfilled composite. J. oral rehabil., Oxford, v. 29, n. 7, p. 644-648, 2002.

ILIE, N.; BUCUTA, S.; DRAENERT, M. Bulk-fill resin-based composites: an in vitro assessment of their mechanical performance. Oper Dent., Seattle, v. 38, n. 6, p. 618-625, 2013.

LEPRINCE, J. G. et al. Physico-mechanical characteristics of commercially available bulk-fill composites. J. Dent., Iran, v. 42, n. 8, p. 993-1000, 2014.

MADEIRA, L. Avaliação da resistência à tração de reparos imediatos em resinas compostas diretas e indiretas. 2002. 83f. Dissertação (Mestrado) - Universidade Federal de Santa Catarina, Florianópolis, 2002.

MATINLINNA, J. P. Silane chemistry aspects in some conventional and novel dental biomaterials. Finlândia: University of Turku, 2004.

OPDAM, N. J. et al. Longevity of posterior composite restorations: a systematic review and meta-analysis. J. dent. res., Washington, v. 93, n.10, p. 943-949, 2014.

OZCAN, M. et al. Repair bond strength of microhybrid, nanohybrid and nanofilled resin composites: effect of substrate resin type, surface conditioning and ageing. Clin. oral investig., Berlin, v.7, n.7, p.17511758, 2013.

RIBEIRO, J. C. V. et al. The microtensile test in bond strength evaluation: fundamentals and applications. Rev. gauch odontol., Porto Alegre, v. 61, p. 497-504, 2013.

RUEGGEBERG, F. A. From vulcanite to vinyl, a history of resins in restorative dentistry. J. prosthet dent., St. Louis, v. 87, n. 4, p. 364-379, Apr. 2002.

TEIXEIRA, C. E. et al. Shear bond strength of self-etching bonding systems in combination with various composites used for repairing aged composites. J. adhes. dent., New Malden, v.7, n. 2, p. 159-164, 2005.

Submetido em: $14 / 11 / 2018$

Aceito em: 29/11/2018 